\title{
Novel research synergized by Chinese medicine pattern classification in rheumatoid arthritis
}

\author{
Chi Zhang ${ }^{1}$ (1) $\cdot$ Aiping Lyu ${ }^{2,3}$ (1)
}

Received: 27 September 2021 / Revised: 30 September 2021 / Accepted: 3 October 2021 / Published online: 9 October 2021

(c) International League of Associations for Rheumatology (ILAR) 2021

\section{What is Chinese medicine pattern classification?}

Pattern classification, the core theory in Chinese medicine clinical practice, is a complementary method to classify an individual's health condition and contribute to personalized medicine [1]. Chinese medicine pattern (CM pattern) entails gathering clinical symptoms and signs through the four diagnostic methods (inspection, listening and smelling, inquiry, and pulse-feeling and palpation) and then classifying an individual's health status to define a specific type of CM pattern [2]. Although CM pattern diagnose (classification) is to identify a stable cluster of possibly concurrent symptoms and signs, the pattern could be dynamic, rather than static. Similar to biomedicine, the collected manifestations are interpreted collectively; however, in the wisdom of Chinese medicine, one disease might display several different " $\mathrm{CM}$ patterns" and be treated by multiple different medications, whereas multiple diseases might share one "CM pattern" and be treated by the same medication. CM pattern classification has already been successfully incorporated with RA diagnosis, resulting in a deepening research interest of $\mathrm{CM}$ pattern among various disciplines of integrative medicine [3].

Aiping Lyu

aipinglu@hkbu.edu.hk

Chi Zhang

saga618@126.com

1 Dongzhimen Hospital, Beijing University of Chinese Medicine, Dongcheng District, Beijing 100700, China

2 School of Chinese Medicine, Hong Kong Baptist University, Kowloon Tong, Kowloon, Hong Kong

3 Institute of Integrated Bioinformedicine \& Translational Science, Hong Kong Baptist University, Kowloon Tong, Kowloon, Hong Kong

\section{What revealed in $\mathrm{CM}$ pattern classification and rheumatoid arthritis?}

To understand the challenges related to the groundbreaking innovation work, it is helpful to review the state of CM pattern classification and RA. In rheumatoid arthritis (RA), the "cold" Pattern ("HAN ZHENG" in Mandarin) and "hot" Pattern ("RE ZHENG" in Mandarin) are the two key statuses of the CM pattern. Those biomedical fundamentals were elucidated by using omics data and bioinformatics approaches [4-6]. Molecular differences between the cold and hot pattern RA groups were found [7]. Additionally, there is clear evidence that the CM pattern classification in RA has shown to be correlated with the clinical efficacy of Tripterygium wilfordii Hook F (TwHF) and classic herbal formula in high-quality clinical trials [8-10]. Interestingly, RA patients with cold pattern had a significantly higher response rate to traditional disease-modifying antirheumatic drugs (DMARDs) than non-cold pattern patients. In addition, the level of C-reactive protein (CRP) discriminated leflunomide responders from non-responders [11]. Subsequently, subgrouping of patients according to the Pattern provides opportunities for improved treatment outcomes [12-14]. Moreover, according to the same Pattern in different diseases, and the similarities among RA, diabetes, and coronary heart disease were preliminarily explored $[15,16]$. These pioneering studies foreshadowed future work demonstrating the importance of $\mathrm{CM}$ pattern, and this approach contributes to a more precise classification for precision medicine.

Recently, many significant efforts have been made to effectively improve the response of old/new agents in the field of RA. Take a DMARD leflunomide (LEF) as an example, in order to be precise, cardiovascular medication ligustrazine (LIG) has been chosen as an addition to the treatment armamentarium based on the same CM pattern. Encouragingly, the LEF-LIG combination shows promising effectiveness for reducing joint inflammation and attenuating bone 
erosion in RA patients [17]. Meanwhile, a classic example of newer tumor necrosis factor (TNF) inhibitor agents has also been explored. TNF inhibitor therapy has revolutionized the treatment of RA. Unfortunately, no one single agent is fully effective in every patient, and different RA patients respond to different therapies in different ways, even to those agents with the same mechanism of action. How can we improve on rates of treatment response? In Chinese medicine, response to treatment may be influenced by the patient's CM pattern dynamic status. Thus, the Pattern dynamic change factor may also be important for the correct selection of medication, but have received little attention to date. In this regard, responders and non-responders of TNF inhibitors in the treatment of RA were analyzed. There are different activation pathways with dynamic change in the pathway (for example, Wnt signaling). While the pathway dynamic change may be helpful in predicting RA drug response differences, the biological pathway usually consists of several genes responsible for diverse functionality. Thus, more finetuned, RA agent and specific, experiments are necessary in order to pinpoint which gene(s) may be the ones most associated with RA agent's sensitivity. Recent study also provides a promising algorithm for revealing potential dynamic mechanism [18]. Now, ongoing animal research aims to test the Pattern dynamic change related to the responses of TNF inhibitor. The importance of delivering the right agents to each RA patient is unambiguously clear. Totally, understanding of the Pattern classification has been expanded and applied for the treatment of RA, which has resulted in the development of more effective therapeutic agents.

\section{So what does the future of $\mathrm{CM}$ pattern classification and RA look like?}

In the near future, CM pattern classification will become more involved in precision medicine regarding scientific investigation, diagnosis, and therapeutic management. Firstly, the fundamentals of the Pattern in a specific disease in biomedical sciences are the key issue to help scientists understand what wisdom is and why it is important to our future researches. The Pattern classification is largely experience-based, but the scientific basis is largely non-satisfactory. Therefore, it is still a great challenge to clarify the action mechanism of the Pattern under the background of biomedical science. Future efforts may include exploration of RA gene-phenotype relationships by integrate gene network and phenotype network, hidden associations between Pattern and the occurrence, development, and treatment response in RA. Furthermore, there is an urgent need to identify key biomarkers or special pathways of non-responders' CM pattern, different activation pathways, and the dynamics of the pathway. Making the Pattern classification clearer will be no mean feat, but the potential benefits are bigger still.

Secondly, big data will continue to grow even bigger in the years to come. Large numbers of CM pattern data are generated through long history medical records. Nowadays, there are excellent platforms to explore the herbal formula-related mechanism, chemical-chemical interactions, herb-chemical drug interactions, optimization of classic herbal formula, ADME, safety assessment, and combination drug discovery. More national registries, such as Chinese Medicine Rheumatology Registration Research Information (CERTAIN), which are linked to the electronic medical record, offer an excellent sampling frame for future prospective studies that could advance the science of the Pattern classification. As mentioned, current technologies produce much more data, such as chemical data, omics data. Those data can and should be used for RA integrative medicine study, with the approaches of bioinformatics, chemoinformatics, and systems pharmacology, to decipher the molecular mechanism of Pattern in RA, the transformation of the Pattern in RA progression with dynamic analysis, and mode of actions of herbal formulas. In brief, big data allows for tailoring than was ever before possible by linking together both the Pattern classification and RA diagnose to reveal hitherto-unknown correlations and causal pathways.

\section{Summary}

It is our opinion that CM pattern classification-related concepts and biomedical backgrounds explored at this particular time of rapid technological change and accretion of knowledge will gain motivation to pursue success in novelty in this area. Zero to one breakthrough is the future by integrating CM pattern classification. By using integrated CM pattern reclassification/transformation/combination and RA biomedical disease diagnosis, a new classification method will gain improved performance over current state-of-the-art disease classification methods. The new integrated method delivers optimal discriminative power for the disease phenotype and finds combination therapy works best for each RA patients.

Funding The National Natural Science Foundation of China (No. 81973688).

\section{Declarations}

Disclosures None. 


\section{References}

1. Lu A, Jiang M, Zhang C, Chan K (2012) An integrative approach of linking traditional Chinese medicine pattern classification and biomedicine diagnosis. J Ethnopharmacol 141:549-556. https:// doi.org/10.1016/j.jep.2011.08.045

2. Jiang M, Lu C, Zhang C, Yang J, Tan Y, Lu A, Chan K (2012) Syndrome differentiation in modern research of traditional Chinese medicine. J Ethnopharmacol 140:634-642. https://doi.org/ 10.1016/j.jep.2012.01.033

3. Zhang C, Zhang G, Chen K, Lu A (2016) Integration of Chinese medicine with Western medicine could lead to future medicine: molecular module medicine. Chin J Integr Med 4:243-250. https://doi.org/10.1007/s11655-016-2495-0

4. Lu C, Xiao C, Chen G, Jiang M, Zha Q, Yan X, Kong W, Lu A (2012) Cold and heat pattern of rheumatoid arthritis in traditional Chinese medicine: distinct molecular signatures indentified by microarray expression profiles in CD4-positive T cell. Rheumatol Int 32:61-68. https://doi.org/10.1007/s00296-010-1546-7

5. Chen G, Lu C, Zha Q, Xiao C, Xu S, Ju D, Zhou Y, Jia W, Lu A (2012) A network-based analysis of traditional Chinese medicine cold and hot patterns in rheumatoid arthritis. Complement Ther Med 20(1-2):23-30. https://doi.org/10.1016/j.ctim.2011.10.005

6. Zhang C, Li L, Zhang G, Chen K, Lu A (2021) Deciphering potential correlations between new biomarkers and pattern classification in Chinese medicine by bioinformatics: two examples of rheumatoid arthritis. Chin J Integr Med 27:465-469. https:// doi.org/10.1007/s11655-018-2571-8

7. van Wietmarschen $\mathrm{H}$, Yuan K, Lu C, Gao P, Wang J, Xiao C, Yan X, Wang M, Schroën J, Lu A, Xu G, van der Greef J (2009) Systems biology guided by Chinese medicine reveals new markers for sub-typing rheumatoid arthritis patients. J Clin Rheumatol 15:330-337. https://doi.org/10.1097/RHU.0b013e3181ba3926

8. He Y, Lu A, Zha Y, Yan X, Song Y, Zeng S, Liu W, Zhu W, Su L, Feng X, Qian X, Lu C (2007) Correlations between symptoms as assessed in traditional Chinese medicine (TCM) and ACR20 efficacy response: a comparison study in 396 patients with rheumatoid arthritis treated with TCM or Western medicine. J Clin Rheumatol 13:317-321. https://doi.org/10.1097/RHU.0b013 e31815d019b

9. He Y, Lu A, Zha Y, Tsang I (2008) Differential effect on symptoms treated with traditional Chinese medicine and western combination therapy in RA patients. Complement Ther Med 16:206211. https://doi.org/10.1016/j.ctim.2007.08.005

10. Jiang M, Zha Q, Lu C, He Y, Lu A (2011) Association between tongue appearance in traditional Chinese medicine and effective response in treatment of rheumatoid arthritis. Complement Ther Med 19:115-121. https://doi.org/10.1016/j.ctim.2011.05.002

11. Liang C, Li J, Lu C, Xie D, Liu J, Zhong C, Wu X, Dai R, Zhang H, Guan D, Guo B, He B, Li F, He X, Zhang W, Zhang B, Zhang
G, Lu A (2019) HIF1 $\alpha$ inhibition facilitates Leflunomide-AHRCRP signaling to attenuate bone erosion in CRP-aberrant rheumatoid arthritis. Nat Commun 10:4579. https://doi.org/10.1038/ s41467-019-12163-z

12 Zhang C, Jiang M, Lu A (2011) A traditional Chinese medicine versus Western combination therapy in the treatment of rheumatoid arthritis: two-stage study protocol for a randomized controlled trial. Trials 12:137. https://doi.org/10.1186/1745-6215-12-137

13. Jiang M, Zha Q, Zhang C, Lu C, Yan X, Zhu W, Liu W, Tu S, Hou L, Wang C, Zhang W, Liang Q, Fan B, Yu J, Zhang W, Liu X, Yang J, He X, Li L, Niu X, Liu Y, Guo H, He B, Zhang G, Bian Z, Lu A (2015) Predicting and verifying outcome of Tripterygium wilfordii Hook F. based therapy in rheumatoid arthritis: from open to double-blinded randomized trial. Sci Rep 5:9700. https://doi. org/10.1038/srep09700

14 Zhang C, Zha QL, He YT, Jiang M, Lu C, Lu AP (2011) The extraarticular symptoms influence ACR response in the treatment of rheumatoid arthritis with biomedicine: a single-blind, randomized, controlled, multicenter trial in 194 patients. J Tradit Chin Med 31:50-55. https://doi.org/10.1016/s0254-6272(11)60012-5

15. Niu X, Lu C, Xiao C, Zhang Z, Jiang M, He D, Bian Y, Zhang G, Bian Z, Lu A (2014) The shared crosstalk of multiple pathways involved in the inflammation between rheumatoid arthritis and coronary artery disease based on a digital gene expression profile. PLoS One 12:e113659. https://doi.org/10.1371/journal.pone. 0113659

16. Niu X, Lu C, Xiao C, Ge N, Jiang M, Li L, Bian Y, Xu G, Bian Z, Zhang G, Lu A (2015) The crosstalk of pathways involved in immune response maybe the shared molecular basis of rheumatoid arthritis and type 2 diabetes. PLoS One 10:e0134990. https://doi. org/10.1371/journal.pone.0134990

17. Zhang C, Guan D, Jiang M, Liang C, Li L, Zhao N, Zha Q, Zhang W, Lu C, Zhang G, Liu J, Lu A (2019) Efficacy of leflunomide combined with ligustrazine in the treatment of rheumatoid arthritis: prediction with network pharmacology and validation in a clinical trial. Chin Med 14:26. https://doi.org/10.1186/ s13020-019-0247-8

18. Zhou W, He X, Chen Z, Fan D, Wang Y, Feng H, Zhang G, Lu A, Xiao L (2019) LncRNA HOTAIR-mediated Wnt/ $\beta$-catenin network modeling to predict and validate therapeutic targets for cartilage damage. BMC Bioinformatics 20:412. https://doi.org/ 10.1186/s12859-019-2981-4

Publisher's note Springer Nature remains neutral with regard to jurisdictional claims in published maps and institutional affiliations. 